\title{
Mitigating Low-Pressure Membrane Fouling by Controlling the Charge of Precipitated Floc Particles
}

\author{
Gregg A. McLeod
}

Greenwood Village, Colorado, USA

\begin{abstract}
Fouling presents the most significant obstacle to optimal low-pressure membrane plant performance. The occurrence of fouling tends to decrease production rates (flux), increase chemical usage incurred during clean-in-place (CIP) process, increase energy costs, shorten membrane life and reduce recovery. Fouling may be of organic or inorganic nature, necessitating more frequent dual chemical cleaning procedures. Regardless of the nature of the foulants, particulate loading onto the membrane fiber surface has been identified as a common mechanism of deteriorating performance. Particulates and colloidal materials such as turbidity, natural organic material (NOM), algae and precipitated coagulant floc accumulate on the membrane surface and disrupt the laminar flow of water through the element. Particulates can either attach or adhere to the membrane surface through electrostatic attraction. One method of reducing this fouling mechanism is to employ controlled coagulation as a direct feed or coupled with a clarification step prior to membrane process. Coagulation can attract and retain naturally occurring particulates and colloidal materials via charge neutralization. Then, by controlling the charge of precipitated floc particulates to align with the surface charge of the membrane element, both types of fouling can be mitigated. This Paper summarizes two demonstrations featuring a pressure feed and a submerged vacuum ultrafiltration (UF) system.
\end{abstract}

Keywords: Coagulant, Floc, Particulate, Flux, Electrostatic.

\section{INTRODUCTION}

Sources of low-pressure membrane fouling may be identified as either organic or inorganic contaminants. Municipal drinking water treatment source waters typically contain a combination of these contaminants including colloidal turbidity, algae, color, various minerals and natural organic material (NOM). Chemical cleanings, including clean-in-place (CIP) or maintenance wash $(\mathrm{MW})$ regimens, generally address inorganic fouling via acid soak/rinse and organic fouling via oxidant soak/rinse.

As regulatory requirements and drinking water standards have tightened, an increasing number of low pressure membrane installations have incorporated enhanced coagulation to increase soluble organic carbon removal [1]. This chemical addition may have variable effects on membrane performance. This paper addresses this practice and outlines a chemical control system to allow coagulant use and limit colloidal and floc particulate fouling potential [2, 3].

A recently described method of controlling the membrane fouling associated with inorganic coagulant precipitation operates under the principle that electrostatics-mediated particulate charge attraction is the primary fouling mechanism at work under these conditions [4]. Managing precipitated floc particle charge can be achieved by guiding a reagent (e.g.

*Address correspondence to this author at the Greenwood Village, Colorado, USA; Tel: 720219 3236; E-mail: gregg@marmac.us.com caustic) dose via a streaming current signal. Streaming current is traditionally used to control coagulant dose for optimum turbidity particle charge mitigation. Enhanced coagulation involves coagulant doses that exceed turbidity charge neutralization, thus rendering the traditional use of streaming current ineffective [5, 6]. However, maintaining a desired particle charge under enhanced coagulation conditions can be achieved by directing reagent feed via a streaming current signal $[7,8]$. The streaming current set point must be within the optimal $\mathrm{pH}$ precipitation range for the specific coagulant $[9,10]$. The addition of anions reduces the cationic burden and minimizes electrostatic attraction or bridging of these particles to a membrane element possessing an anionic surface charge [11].

Some of the earliest microfiltration (MF) drinking water installations began dosing a chemical coagulant ahead of their membrane systems for true (soluble) color removal. Inorganic coagulants are effective at higher molecular weight NOM removal [12]. Aluminum chlorhydrate $(\mathrm{ACH})$ and polyaluminum chloride $(\mathrm{PACl})$ were initially employed for their effectiveness at removing true color [13]. In some applications, a significant decline in fouling rate was observed. This led some to designate $\mathrm{ACH}$ and $\mathrm{PACl}$ as "membranefriendly" coagulants. However, the effect was not universal, as some installations experienced the reverse effect or an increase in fouling. Operational pressure to achieve higher soluble organic levels (DBP) led many facilities to incorporate more acidic coagulants such as aluminum sulfate or ferric chloride. Coagulant selection was determined based on superior 
soluble organic removal coinciding with a higher proportion of plants experiencing significant increase in fouling [14]. Problematic performance eliminated these coagulants for consideration. However, these results are not universal and do not explain the minority of installations that experience improved membrane performance. Accelerated fouling is more common at facilities employing direct coagulant feed. The majority of MF/UF installations in North America use direct coagulant feed. Pretreatment design to include clarification will reduce this fouling rate by limiting particulate contact with membrane elements, but at a higher capital cost $[15,16]$. Listing these coagulants by historical performance from best to worst $-\mathrm{ACH}, \mathrm{PACl}$, Alum, Ferric Chloride -incidentally ranks the coagulants by their acidity (ACH - least acidic; Ferric Chloride - most acidic). A more acidic coagulant will impart a greater cationic charge at the same dose as a less acidic product. The final particle charge has a greater influence on membrane performance than coagulant selection.

Coagulant selection preferences appear to have originated in shared experiences treating surface waters with similar average raw water $\mathrm{pH}$ and alkalinity. The minimally acidic coagulant, $\mathrm{ACH}$ precipitates at a higher $\mathrm{pH}$ and demonstrates good membrane performance under the conditions seen at a majority of installations. Ferric chloride generally exhibits the poorest membrane performance; its acidity does not optimize the $\mathrm{pH}$ and average particle charge of most surface waters for which it is employed to treat. Nevertheless, at installations where raw water $\mathrm{pH}$, alkalinity and coagulant dose align favorably, higher performance than anticipated may be observed. This paper specifically addresses particulate fouling associated with enhanced chemical coagulant feed.

\section{METHODS AND MATERIALS}

This paper highlights two separate demonstrations involving controlled coagulant feed ahead of lowpressure membrane filtration on a direct feed configuration (no clarification or sedimentation pretreatment). Case Study \#1 involved a $30 \mathrm{mgd}$ submerged vacuum UF system located in Berthoud, Colorado; USA. The demonstration utilized a separate $50 \mathrm{gpm}$ four (4) module pilot unit. The pilot unit was installed at the Berthoud facility utilizing the same feed water as the existing system. In order to ensure comparative results, four (4) modules from the existing system were pulled and installed in the pilot unit. Piping to the pilot simulated existing plant detention time and mixing. Coagulant selection (aluminum chlorhydrate), dose and UF flux also emulated existing plant conditions. Pilot operating data including flux, $\mathrm{pH}$, temperature, resistance and permeability were data logged and compared with operation of the existing facility in order to more fairly compare results of the two systems.

Case Study \#2 involved a 2 mgd pressure UF system located in Idaho Springs, Colorado; USA. The demonstration was conducted "full scale". The existing system utilizes two (2) $\times 0.9$ mgd pressure UF skids. Polyaluminum chloride coagulant was dosed "in-line" upstream of both skids. Controlled chemical feed involving liquid caustic soda regulated via a streaming current signal was performed on skid \#2 only. The results would demonstrate an actual "side by side" comparison of controlled versus uncontrolled feed. Both skids operated at the same flux value. Full scale operating data included flux, $\mathrm{pH}$, temperature, resistance and permeability.

\section{DISCUSSION}

The principle of electrostatics underlies the membrane performance of these coagulants. Acidic coagulants precipitate as positively-charged particles after anionic charges in the water are neutralized. Electrostatic attraction results in adsorption of precipitates to negatively-charged membrane elements. Modification of the charge differential between precipitates and the surface of the membrane element reduces particle adsorption and subsequent fouling $[17,18]$.

Controlling a reagent feed, e.g. caustic, via streaming current signal decreases particle accumulation on membrane elements, significantly reducing trans-membrane pressure. The benefits of optimizing particle charge to reduce fouling by electrostatic attraction can be achieved with any coagulant through $\mathrm{pH}$ modification with temperature compensation. Several demonstrations have been performed, including two highlighted below, in which controlled coagulant dosing achieved a dramatic reduction in low-pressure membrane fouling.

An increasing number of low-pressure membrane installations in the United States employ coagulation and enhanced coagulation as a means of complying with the Stage 1 and Stage 2 Disinfection By Product Rules (DBPR) established by the Environmental Protection Agency in 1998 and 2006, respectively [19]. 
However, high coagulant doses generate precipitated particles with stronger cationic charge when neutralization by natural anionic charges (e.g. humic acids) in the feed water is exceeded, resulting in electrostatic attraction-mediated membrane fouling. A balance must be achieved between the excess of cationic charges introduced by doses involved in enhanced coagulation and the combined anionic reservoir of natural turbidity burden plus anions supplemented by a calculated caustic dose [20]. This balance minimizes charged particulate fouling and trans-membrane pressure and increases installation efficiency by permitting longer filter run times between CIPs and MWs [21].

\section{EXPERIMENTAL}

\section{Case Study Examples}

\section{Case Study \#1}

An existing 30 million-gallon per day (mgd) submerged vacuum UF system located in Colorado; USA has been operating for the past 7 years. The plant doses $8-14$ ppm aluminum chlorhydrate $(\mathrm{ACH})$ in a direct feed ahead of the membrane filters. The existing plant does not employ pretreatment ahead of the UF system. Coagulant is dosed ahead of an in-line jet mixer prior to the UF cells. ACH is successful in reducing soluble organic removal in the range of $35-$ $40 \%$, which is sufficient for Disinfection by Product regulatory compliance. Unfortunately, unacceptable fouling limits the plant production to $20 \mathrm{mgd}$.

The treatment facility owner, working with their engineer initiated a demonstration study to test a 50 gallon per minute (gpm) pilot system provided by the membrane process manufacturer. Membrane elements were retrieved from the existing plant to accurately compare performance of similar operation (coagulant dose, detention time etc.) versus a controlled feed. Coagulant and caustic are dosed in-line prior to a static mixer with pipe run to emulate plant detention time.

Both plant and pilot maintained $12 \mathrm{ppm} \mathrm{ACH}$ coagulant dose. Baseline runs emulating existing plant performance provided similar results in transmembrane and resistance performance. Particulate accumulation, especially at the upper portion of the membrane module matched that of the existing plant (See Figure 1). During these baseline runs, a correlation appeared between this particulate accumulation and TMP rise. During initial controlled trial, starting filter TMP value was recorded 1 minute into each run to gauge differential in fouling rate. Similar to baseline run, a correlation was observed between reduction in particulate accumulation and TMP stabilization (See Figure 2).

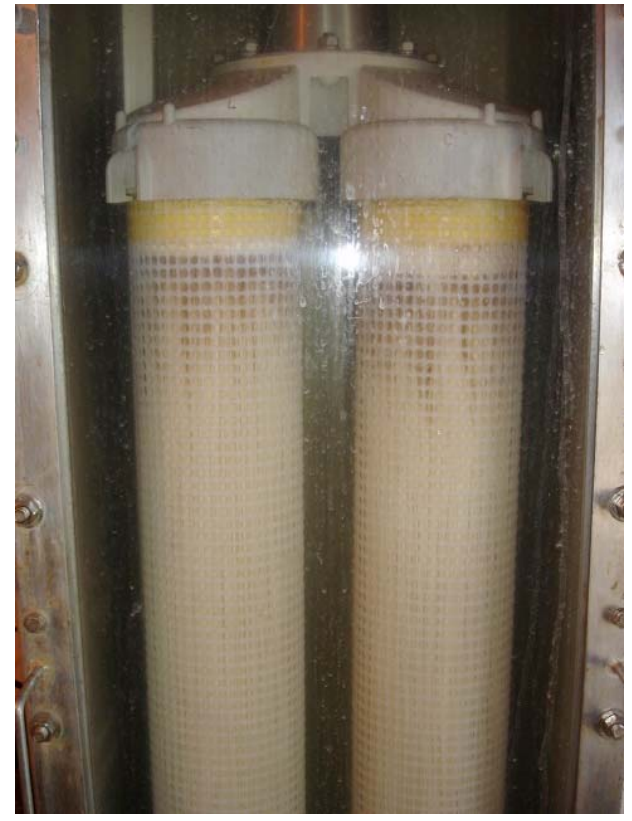

Figure 1: Flux: $36 \mathrm{gfd}$; ACH Dose: $12 \mathrm{ppm}$; Run time: 14 days Visible particle accumulation on membrane elements.

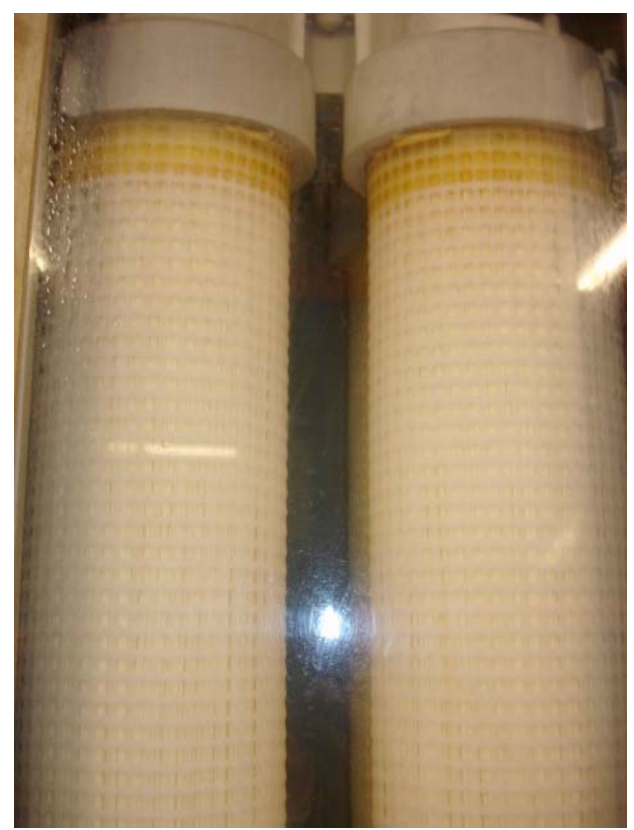

Figure 2: Flux: $36 \mathrm{gfd}$; $\mathrm{ACH}$ Dose: $12 \mathrm{ppm}$; Run time: 14 days No visible particle accumulation on elements.

The pilot performance comparison clearly demonstrated a reduction in TMP rise and particulate accumulation for controlled versus uncontrolled coagulant feed. TMP rise for the control run decreased by $60 \%$ versus baseline (See Figures 3 and 4 ). 


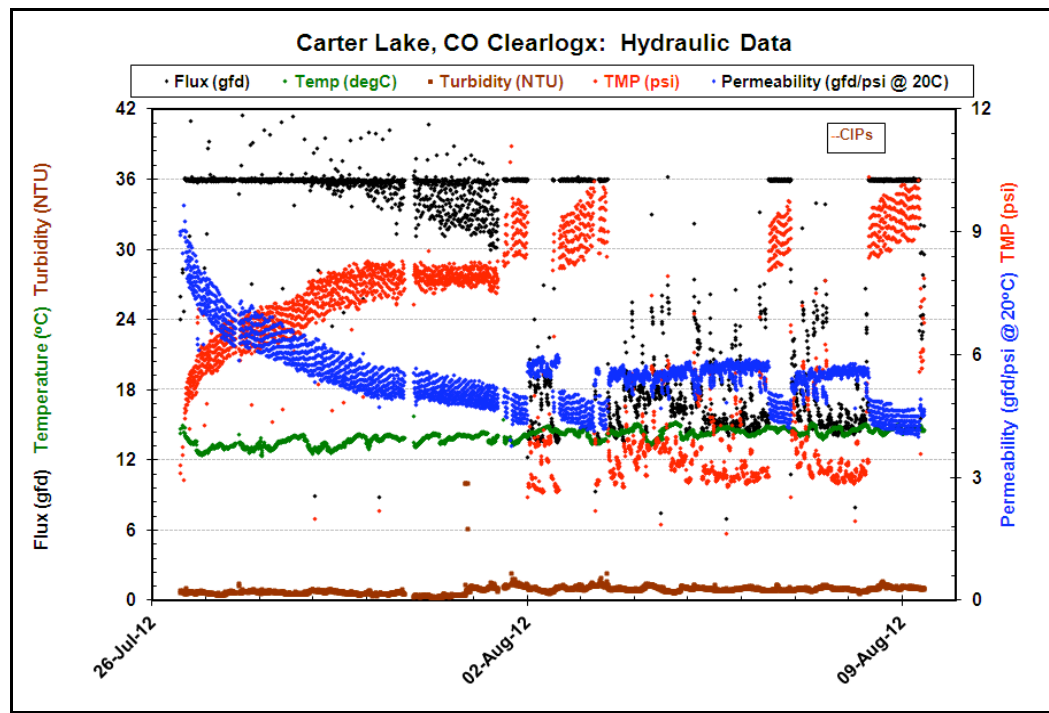

Figure 3: Baseline Pilot Run without $\mathrm{pH} /$ particle charge control. TMP rise (Red): 1.3 psi/day.

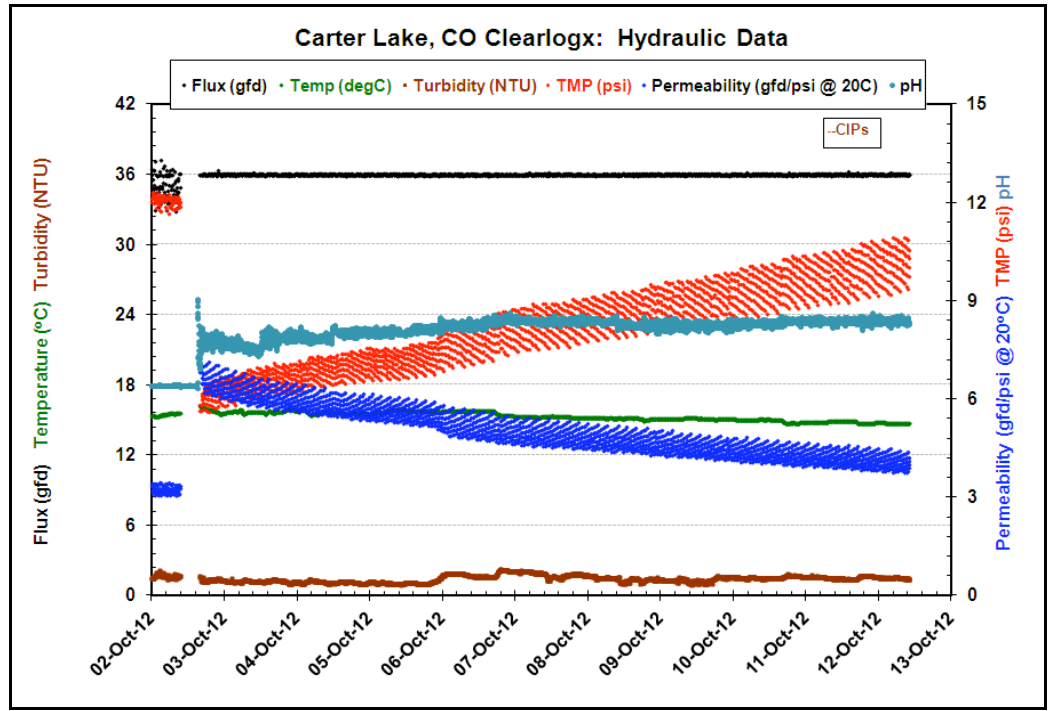

Figure 4: Control Pilot Run with $\mathrm{pH} /$ particle charge control. TMP rise (Red): $0.38 \mathrm{psi} / \mathrm{day}$.

\section{Case Study \#2}

An existing 2 mgd pressure UF system located in Colorado, USA has been operating since 2004. The plant achieved acceptable performance and regulatory compliance without the requirement for coagulant addition. When faced with tighter disinfection by product (DBP) regulatory limits in 2012, the district engineer recommended enhanced coagulation to improve soluble organic removal. Polyaluminum chloride (PACl) was recommended as the preferred coagulant. The plant initiated direct $20 \mathrm{ppm}$ coagulant feed dosing ahead of the membrane units (no sedimentation or clarification). The plant noticed an immediate increase in TMP and decrease in permeability. The increase in fouling necessitated suspension of coagulant addition. A proprietary chemical feed control system was recommended (Clearlog $\mathrm{x}^{\mathrm{TM}}$ ) as a means of permitting direct $\mathrm{PACl}$ coagulant feed while minimizing fouling. The chemical feed system operates on the principles described within, controlling liquid caustic feed via streaming current signal to maintain optimum particle charge value. In order to compare results on a full-scale basis, the engineer recommended an uncontrolled feed on skid \#1 and a controlled feed on skid \#2. Both skids received the same $20 \mathrm{ppm}$ coagulant dose via a single injection upstream of the units. The chemical feed system regulated caustic feed ahead of skid \#2. Skid \#1 repeated previous uncontrolled dose results depicting rapid fouling (Figure 5). Skid \#2 demonstrated minimal TMP increase and permeability decline (Figure 6). 


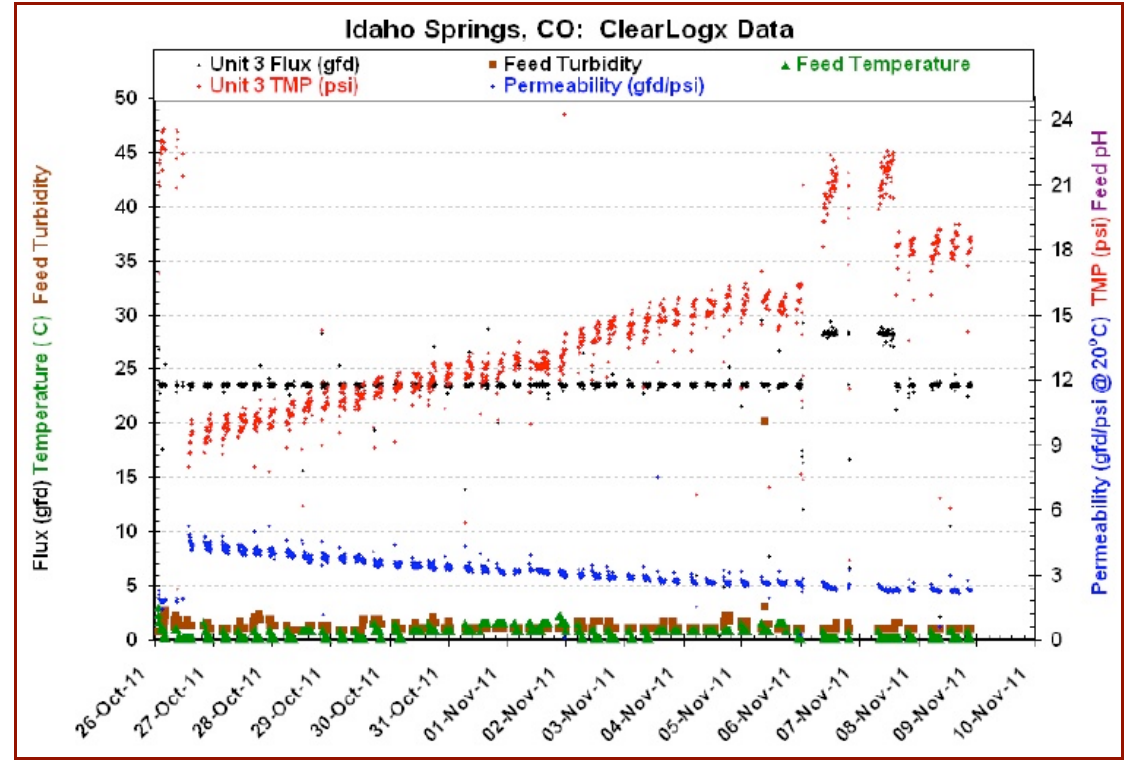

Figure 5: Skid \#1; 20 ppm Uncontrolled PACl Dose. TMP Rise = 6 psi/day.

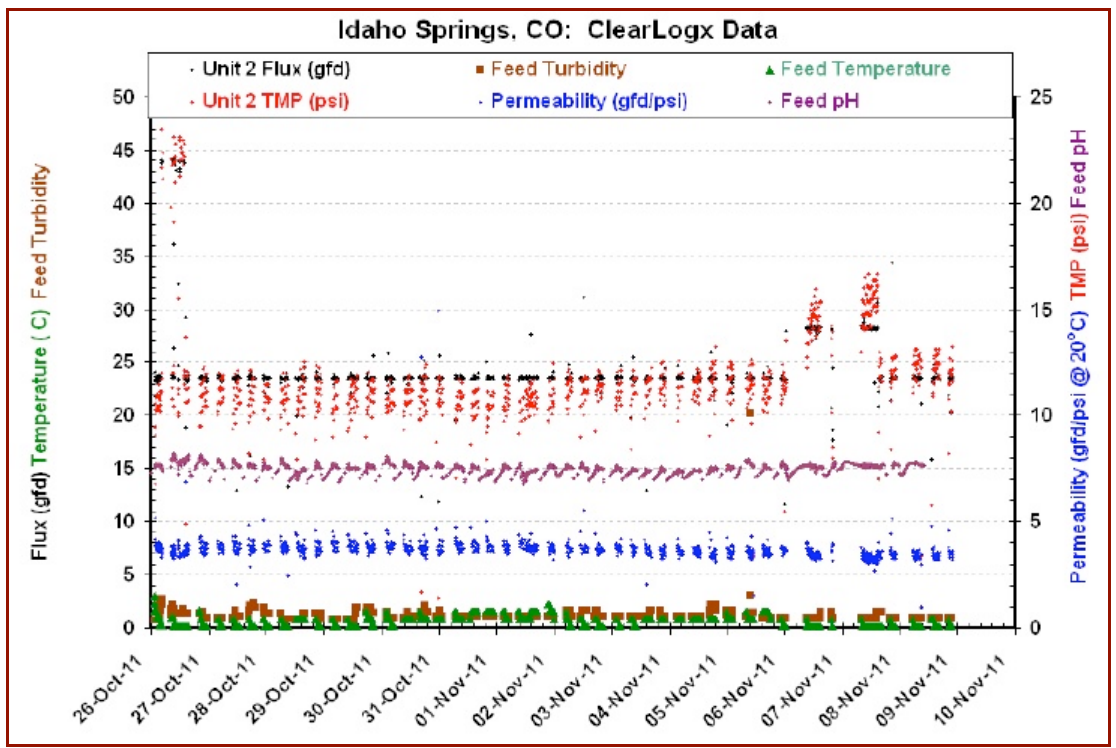

Figure 6: Skid \#2; 20 ppm Controlled PACI Dose. TMP Rise $=<0.1 \mathrm{psi} /$ day.

Figures 5 and 6 demonstrate a clear difference in operating performance in terms of comparative TMP and Permeability at identical flux. Full scale pilot demonstrations on pressure type module-canister housings preclude visual observation of particulate accumulation. Trial results are dependent on indicative fouling measurement monitoring TMP and Permeability over time. The uncontrolled baseline fouling rate when compared to Case Study \#1 herein is considerably higher at lower flux. Both facilities utilize similar surface water source quality in terms of organic load, turbidity, $\mathrm{pH}$ and alkalinity. Coagulant dose is higher for this demonstration utilizing a slightly more acidic product which can account for this differential.

\section{CONCLUSIONS}

The result of these demonstrations is significant in terms of capital and operating cost savings. Both facilities highlighted were facing costly membrane expansion with an associated increase in operating costs in order to offset fouling associated with enhanced coagulation. After the conclusion of this testing, both facilities incorporated chemical dosing automation and controls and have maintained regulatory compliance while simultaneously improving membrane filter performance. The ability to achieve similar reductions in membrane fouling can benefit a large number of existing and future filtration facilities. Recognized fouling mechanisms such as inorganic and 
organic fouling should include electrostatic attractionmediated particulate fouling, particularly in the context of enhanced coagulation. Understanding the chemical characteristics of feed water quality, chemical feed and membrane material allows an operator to minimize this major component of fouling, resulting in a highly efficient membrane filtration system. Additional automated controls can potentially offset against feed water changes relating to snow melt, rain events, drought conditions and changing water supplies. The investment in proper controls and automation may not only improve membrane performance but also provide a rapid return on this investment.

\section{REFERENCES}

[1] de la Rubia A, Rodriguez M, Leon V M, et al. Removal of natural organic matter and THM formation potential by ultraand nanofiltration- of surface water. Water Res 2008; 42: 714-722. http://dx.doi.org/10.1016/j.watres.2007.07.049

[2] Lee $\mathrm{N}$, Amy $\mathrm{G}$, Croue $\mathrm{J}$, et al. Identification and understanding of fouling in low pressure Membrane (MF/UF) filtration by natural organic matter (NOM). Water Res 2004; 38: 4511-4523.

http://dx.doi.org/10.1016/j.watres.2004.08.013

[3] Jermann D, Pronk W, Kagi R, et al. Influence of interactions between NOM and particles inUF fouling mechanisms. Water Res 2008; 42: 3870-3878. http://dx.doi.org/10.1016/j.watres.2008.05.013

[4] Mcleod GM, Inventor "Process for enhanced total organic carbon removal while maintaining optimum membrane filter performance" United States Patent 8,540,882B2; 2013 Sep; 8,491,794B2; 2013 Sep.

[5] Sharp E, Ratnaweera. Treatment of waters with elevated organic content. AWWA Journal 2006.

[6] Kim S, Moon S, Yoon C. Identification of fouling causing materials in the ultrafiltration of surface water. Desalination 2005; 177: 201-207.

http://dx.doi.org/10.1016/j.desal.2004.11.020

[7] Briley, D, Knappe Detlef R. Streaming current coagulation. AWWA Journal Feb 2002.

[8] Walker C, Kirby J, Dental S. Streaming current detector: a quantitative model. Journal of Colloid and Interface Science March 19960438.

[9] Parthasarathy N, Buffle J. Study of polymeric aluminum (III) hydroxide solutions for application in waste water treatment.
Properties of the polymer and optimal conditions of preparation. Water Res 1991; 25-36.

[10] Bertsch P, Thomas G. Characterization of hydroxyl aluminum solutions by aluminum 27 nuclear magnetic resonance spectroscopy. Soil Science Society of America Journal 50 : 825-830. http://dx.doi.org/10.2136/sssaj1986.03615995005000030051 $\underline{x}$

[11] Jermann D, Pronk W, Kagi R, et al. Influence of interactions between NOM and particles in UF fouling mechanisms. Water Res 2008; 42: 3870-3878.

http://dx.doi.org/10.1016/j.watres.2008.05.013

[12] Li Yon Hong, Wang Jun, Zhang Wei, Zhang XiioJian, Chen Chao. Effects of coagulation on submerged ultrafiltration membrane fouling caused by particles and natural organic matter (NOM). Chinese Science Bulletin 2011; 56: 584-590. http://dx.doi.org/10.1007/s11434-010-4296-8

[13] Pernitsky D. Coagulation 101. AWWA 1987.

[14] Van Benschoten J, Edzwald J. Chemical aspects of coagulation using aluminum salts. Hydrolytic reactions of alum and polyaluminum chloride. Water Res 24(12): 15191526.

http://dx.doi.org/10.1016/0043-1354(90)90086-L

[15] Huang H, Schwab K, Jacangelo J. Pretreatment for low pressure membranes in water treatment: $A$ review. Environ Sci Techno 2009; 43: 3011-3019. http://dx.doi.org/10.1021/es802473r

[16] Maartens A, Swart P, Jacobs E. Feed water pretreatment: methods to reduce membrane fouling by natural organic matter. Journal of Membrane Science 1999; 163: 51-62. http://dx.doi.org/10.1016/S0376-7388(99)00155-6

[17] Li Y, Zhang W, Zhang X, et al. Characterization of fouling in immersed polyvinylidene fluoride hollow fiber membrane ultrafiltration by particles and natural organic matter (NOM). Chinese Science Bulletin 2011; 56: 584-590. http://dx.doi.org/10.1007/s11434-010-4296-8

[18] Chen $\mathrm{Y}$, Dong $\mathrm{B}$, Gao $\mathrm{N}$, et al. Effect of coagulation pretreatment on fouling of an ultrafiltration membrane. Desalination 2007; 204: 181-188. http://dx.doi.org/10.1016/j.desal.2006.04.029

[19] United States EPA National primary drinking water regulations; disinfectants and disinfection by products. EPA 816-F-02-021 Stage 1; Dec 16, 1998. EPA 815-F-05-003 Stage 2; Jan 4, 2006.

[20] Kim, S, Moon S, Yoon C. Identification of fouling causing materials in the ultrafiltration of surface water. Desalination 2005; 177: 201-207. http://dx.doi.org/10.1016/j.desal.2004.11.020

[21] Boerlag S, Kennedy M, Bonne $P$, et al. Prediction of flux decline in membrane systems due to particulate fouling. Desalination 1997; 113: 231-233. http://dx.doi.org/10.1016/S0011-9164(97)00134-3 